\title{
Quality of life in a phase 2 trial of short-course hyperthermic intraperitoneal chemotherapy (HIPEC) at interval debulking surgery for high tumor burden ovarian cancer
}

\author{
Avaliação da qualidade de vida em ensaio clínico de fase 2 envolvendo \\ quimioterapia intraperitoneal hipertérmica (HIPEC) de curta duração no momen- \\ to da citorredução de intervalo em pacientes com câncer de ovário de grande \\ volume tumoral
}

Roberto José Costa lustosa 1 ili ; Thales Paulo Batista, TCBC-Pe²; Vandré Cabral Gomes Carneiro3; Levon Badiglan-Filho4; Ronaldo lúcio Rangel Costa5; André lopes5; Bruno José de Queiroz Sarmento, TCBC-DF6; Jurema Telles de Oliveira Lima7; Maria Julia Gonçalves de Mello8; Cristiano Souza leão'.

\begin{abstract}
A B S T R A C T
Introduction: to evaluate the effect of short-course (i.e.: 30 minutes) HIPEC on health-related quality of life (HRQoL) in our feasibility study; NCT02249013. Methods: a prespecified secondary end-point of our open-label, multicenter, single-arm, phase 2 trial on safety and efficacy was assessed using the European Organization for Research and Treatment of Cancer Quality of Life Questionnaire Core 30 (EORTC QLQ-C30, version 3.0). Patients were required to complete the HRQoL questionnaire at baseline, after HIPEC, and after the end of the treatment. Changes of HRQoL over time were assessed by median scores for each domain and analyzed by Friedman's test at a significant two-sided level of 0.05. Results: fifteen patients with high tumor burden EOC were recruited from our public health system between February 2015 and July 2019. A baseline EORTC QLQ-C30 questionnaire and at least one follow-up questionnaire was received from all of the patients. No significant difference over time in the QLQC30 summary scores was observed ( $p>0.05)$. The transitory impairment on patients HRQoL immediately after the short-course HIPEC trended to return to baseline at the end of the multimodal treatment. Conclusions: we found no significant impairment of short-course HIPEC on patients HRQoL into the context of our comprehensive treatment protocol.
\end{abstract}

Keywords: Injections Intraperitoneal. Hyperthermia, Induced. Drug Therapy. Peritoneal Neoplasms. Surgical Procedures, Operative.

\section{INTRODUCTION}

E pithelial ovarian cancer (EOC) is a challenging disease that tends to be diagnosed in advanced stages and disseminated into the peritoneal cavity, making the treatment of peritoneum a cornerstone in the management of this gynecologic malignancy. In these settings, intraperitoneal (IP) chemotherapy continue to be strongly supported for optimally debulked patients, particularly those with no gross residual disease and those with BRCA mutations or other manifestations of homologous repair deficiency'. However, this approach has not been widely adopted in the clinical practice mainly due to higher toxicity, inconvenience and catheter-related complications ${ }^{2,3}$, as well as to impairment on quality of life (QoL) when compared with patients receiving

1 - Departamento de Cirurgia, IMIP - Instituto de Medicina Integral Professor Fernando Figueira; Recife - PE, Brasil 2 - Departamento de Cirurgia I Oncologia, IMIP - Instituto de Medicina Integral Professor Fernando Figueira; e Departamento de Cirurgia, UFPE - Universidade Federal de Pernambuco; Recife - PE, Brasil 3 - Departamento de Cirurgia / Oncologia, IMIP - Instituto de Medicina Integral Professor Fernando Figueira Departamento de Cirurgia; e do Departamento de Ginecologia, HCP - Hospital de Câncer de Pernambuco; Recife - PE, Brasil 4 - Departamento de Ginecologia, AC Camargo Cancer Center; São Paulo - SP, Brasil 5 - Departamento de Ginecologia, IBCC - Instituto Brasileiro de Controle do Câncer, São Paulo - SP, Brasil 6 - Departamento de Oncologia Cirúrgica, IHBDF - Instituto Hospital de Base do Distrito Federal, Brasília - DF, Brasil 7 - Departamento de Oncologia Clínica, IMIP - Instituto de Medicina Integral Professor Fernando Figueira; Recife - PE, Brasil 8 - Departamento de Pesquisa Clínica, IMIP - Instituto de Medicina Integral Professor Fernando Figueira; Recife - PE, Brasil 
conventional systemic chemotherapy alone ${ }^{4}$.

Hyperthermic intraperitoneal chemotherapy (HIPEC) has been presented as a promising alternative to IP route for chemotherapy in EOC ${ }^{5}$. In this approach, chemotherapy is delivered intraoperatively, avoiding the need for implantation of peritoneal access devices and thereby reducing catheter-related morbidity and negating the issues of tolerance. Recently, the OV-HIPEC trial by Van Driel et al. ${ }^{6}$ reported benefits in both progression-free and overall survival in favor of HIPEC at the time of interval debulking surgery (IDS) for high tumor burden patients not eligible for primary debulking surgery. The effect of HIPEC on patient's health-related QoL (HRQoL) was assessed in this trial using the European Organization for Research and Treatment of Cancer (EORTC) QoL Questionnaire Core 30 (QLQ-C30), the ovarian (QLQ-OV28) and colorectal cancer (QLQ-CR38) modules, which demonstrated the addition of HIPEC to IDS does not negatively impact HRQoL'? Thereafter, an analysis of OV-HIPEC trial also indicated the cost-effective benefit of HIPEC in this patient population8. Despite some criticism 9.10 , HIPEC has emerged as a main comprehensive treatment for advanced EOC, and now can be advocated as a treatment option based on data from a randomized controlled trial ${ }^{6}$.

Herein, we report early outcomes in terms of HRQoL from our open-label, multicenter, single-arm, phase 2 trial. This feasibility study explored the safety and efficacy of a short-course HIPEC protocol under the hypothesis of low morbidity rates, reduced impairment on patient's HRQoL, and improved PD9 (i.e.: proportion of patients with disease progression or death occurring within 9 months of IDS plus HIPEC) ${ }^{11}$

\section{METHODS}

This is a prespecified analysis of the secondary end-point on HRQoL from our phase 2 trial exploring short-course (i.e.: 30 minutes) HIPEC at the time of IDS following neoadjuvant chemotherapy (NACT) for high tumor burden EOC. As part of data collection, patients were required to complete a HRQoL questionnaire at baseline just before the IDS/HIPEC procedure (i.e.: at the time of hospital admission), and repeated after the IDS/HIPEC (i.e.: at the time of re-stating the systemic chemotherapy) and after completion of the treatment protocol (i.e.: at 3-6 weeks after the last systemic chemotherapy cycle).

Details of the study design were previously reported5 and is registered at ClinicalTrials.gov under the identifier NCT02249013. In summary, patients with a high tumour burden were assigned to NACT followed by fast-track IDS plus short-course HIPEC, then postoperative chemotherapy. Systemic chemotherapy was scheduled in the total of 6 perioperative cycles of the standard combination of carboplatin (AUC 6) and paclitaxel (175 $\mathrm{mg} / \mathrm{m} 2$ ) administered every 21 days, adopting the usual criteria for dose modification or delay, as appropriated. HIPEC was performed according to the closed-abdomen technique using cisplatin ( $25 \mathrm{mg} / \mathrm{L}$ of perfusate $/ \mathrm{m} 2$, total limit of $240 \mathrm{mg}$ ) or cisplatin plus Doxorubicin $(15 \mathrm{mg} / \mathrm{L})$ in 4-6L of perfusate for 30 minutes with an intra-abdominal target temperature of $41-43^{\circ} \mathrm{C}$.

HRQoL was assessed with the EORTC QLQ-C30 (version 3.0, Brazilian Portuguese). The QLQ-C30 is a 30item questionnaire that includes five multiitem functional scales (physical, role, cognitive, emotional, and social), three multiitem symptom scales (fatigue, nausea and vomiting, and pain), five single-item symptom scales (dyspnea, insomnia, appetite loss, constipation, and diarrhea), a question on financial impact; and a two-item global QoL scale ${ }^{12}$.

The scales and items of the questionnaires were linearly transformed and analyzed according to the EORTC QoL group procedures ${ }^{12}$. Accordingly, higher scores on the functioning scales and the global health status scale indicate higher level of functioning and better QoL, whereas higher scores on symptoms correspond to worse or more symptoms. Random missing values within a subscale were replaced by the average score of the completed items in the same scale for each individual, provided that at least $50 \%$ of the items in that scale had been completed. For single missing questionnaires, there were no imputation considerations. Changes of HRQoL over time were assessed by median (IQR, interquartile range) scores for each domain and analyzed by Friedman's test at a significant two-sided level of 0.05 .

The study protocol was reviewed by our Ethics Research Committees (Reference No.: CAAE 18388113.4.0000.5201, acceptance protocol at the coordinator center No.: 672.484; May 26, 2014) and thus 
registered on ClinicalTrials.gov (NCT02249013). Written informed consent was obtained from all patients and the procedures complied with the standards of current ethical guidelines. Funding sources were from Decit/ SCTIE/MS - CNPq/FACEPE/SES-PE (APQ:0187-4.01/13) and FAPE/IMIP.

\section{RESULTS}

Fifteen patients with bulky stage III who underwent NACT were recruited from our Public Health System named SUS (i.e.: Sistema Único de Saúde) and allocated to short-course HIPEC between July 2015 and July 2019. The median (IQR) age of patients was 46 years (42$59.5)$, with preoperative serum CA125 levels of $765.4 \mathrm{U} / \mathrm{mL}$ (514.4-1530.2). The median number of NACT cycles was 3 (3-4), resulting in $\mathrm{PCl}$ scores of $11(8-15.5)$ at the time of IDS/HIPEC - performed after 30 days (27.5-32) from the last NACT cycle. Fourteen patients completed all the six cycles of intravenous chemotherapy as planned, with a median time to start adjuvant chemotherapy of 39 days (35-49.3).

Median operation time was 490 minutes (390605.8) and no ostomies were performed in the cohort, despite nine patients requiring major bowel resection as rectosigmoidectomy $(n=8)$ or partial colectomy $(n=1)$. Length of hospital stay was five days (4-6.5), with a median ICU stay of one day (1-1). Four patients experienced no postoperative complications, whereas five suffered only minor G1/G2 adverse effects, and six suffered major G3 adverse effects, according to the NCI/CTCAE Version 4.0 classification. Two patients underwent reoperation because of G3 postoperative hemorrhage or peritoneal infection, respectively; whereas no deaths were recorded.

A baseline EORTC QLQ-C30 questionnaire and at least one follow-up questionnaire was received from all of the patients. Thirteen (86.7\%) patients completed questionnaires after HIPEC, and eleven (73.3\%) responses were received after the completeness of the treatment protocol. Median time to respond the questionnaires were 32 (25-42) and 52 (31.5-92.5) days, respectively. The second time point questionnaire was not collected from two patients that started adjuvant chemotherapy before investigators' assessment. Two patients were not able to complete the last questionnaire due to disease progression or treatment of postoperative complications, respectively. Other reason for not collecting responses after the end of protocol was intermittent loss of follow-up due to patients' reasons in two cases.

No significant difference over time in the QLQ-C30 summary scores was observed ( $p>0.05$ ). The transitory impairment on patient's HRQoL immediately after short-course HIPEC trended to return to baseline at the end of the adjuvant treatment. The EORTC QLQ-C30 functioning and symptom scales at different time points in our trial are summarized in Tables 1 and 2, respectively.

Table 1. EORTC QLQ-C3O scores over time according to functioning scales of patients with ovarian cancer submitted to short-course hyperthermic intraperitoneal chemotherapy (HIPEC).

\begin{tabular}{|c|c|c|c|c|}
\hline \multirow[b]{2}{*}{ EORTC Functioning Scales } & \multicolumn{3}{|c|}{ Questionnaire Timepoints $^{1}$} & \multirow[b]{2}{*}{$p$-value } \\
\hline & Baseline & After HIPEC & After Protocol & \\
\hline Physical functioning & $86.7(80-100)$ & $66.7(66.7-80)$ & $86.7(76.7-90)$ & 0.066 \\
\hline Role functioning & $100.0(50-100)$ & $50.0(16.7-66.7)$ & $100.0(75-100)$ & 0.055 \\
\hline Emotional functioning & $75.0(33.3-87.5)$ & $58.3(41.7-91.7)$ & $66.7(38.9-91.7)$ & 0.541 \\
\hline Cognitive functioning & $83.3(66.7-100)$ & $83.3(50-83.3)$ & $83.3(58.3-100)$ & 0.963 \\
\hline Social functioning & $83.3(58.3-100)$ & $66.7(33.3-100)$ & $83.3(58.3-100)$ & 0.564 \\
\hline Global health status & $83.3(75-100)$ & $66.7(58.3-83.3)$ & $83.3(62.5-91.7)$ & 0.295 \\
\hline
\end{tabular}

${ }^{1}$ Patients were required to complete the questionnaire at baseline, after HIPEC, and after the end of the treatment protocol. All subscale responses were converted to 0-100 scales, according to the EORTC guidelines. Changes over time were assessed by median (IQR, interquartile range) scores for each domain.

${ }^{2}$ Analyzed by Friedman's test at a significant two-sided level of 0.05 . 
Table 2. EORTC QLQC-30 scores over time according to symptom scales of patients with ovarian cancer submitted to short-course hyperthermic intraperitoneal chemotherapy (HIPEC).

\begin{tabular}{lcccc}
\hline & \multicolumn{3}{c}{ Questionnaire Timepoints } & \\
\cline { 2 - 3 } EORTC Symptom Scales & Baseline & After HIPEC & After Protocol & p-value ${ }^{2}$ \\
\hline Fatigue & $22.2(0-27.8)$ & $33.3(33.3-44.4)$ & $33.3(0-38.9)$ & 0.065 \\
Nausea and vomiting & $0.0(0-16.7)$ & $16.7(0-16.7)$ & $0.0(0-16.7)$ & 0.320 \\
Pain & $0.0(0-33.3)$ & $16.7(0-50)$ & $16.7(0-50)$ & 0.525 \\
Dyspnoea & $0.0(0-0)$ & $0.0(0-0)$ & $0.0(0-0)$ & 0.367 \\
Insomnia & $0.0(0-33.3)$ & $33.3(0-66.7)$ & $0.0(0-50)$ & 0.411 \\
Appetite loss & $0.0(0-0)$ & $33.3(0-33.3)$ & $0.0(0-0)$ & 0.311 \\
Constipation & $0.0(0-33.3)$ & $0.0(0-66.7)$ & $33.3(16.7-66.7)$ & 0.778 \\
Diarrhea & $0.0(0-0)$ & $0.0(0-0)$ & $0.0(0-0)$ & 0.716 \\
Financial difficulties & $33.3(0-67.7)$ & $33.3(0-100)$ & $33.3(0-50)$ & 1.000 \\
Diarrhea & $0.0(0-0)$ & $0.0(0-0)$ & $0.0(0-0)$ & 0.716
\end{tabular}

'Patients were required to complete the questionnaire at baseline, after HIPEC, and after the end of the treatment protocol. All subscale responses were converted to 0-100 scales, according to the EORTC guidelines. Changes over time were assessed by median (IQR, interquartile range) scores for each domain.

${ }^{2}$ Analyzed by Friedman's test at a significant two-sided level of 0.05.

\section{DISCUSSION}

Primary debulking surgery followed by a systemic therapy is the mainstay of treatment for advanced EOC ${ }^{13}$. However, HIPEC has emerged as a main comprehensive treatment option for high tumor burden patients based on long-term survival advantages of IP chemotherapy ${ }^{14}$ and recent data from OV-HIPEC trial by Van Driel et al. ${ }^{6}$ In these settings, we present early outcomes in terms of HRQoL from our feasibility study, which explore the potential advantages of combining the early intraperitoneal route for chemotherapy without the need for abdominal catheters, the synergism of hyperthermia, and the benefits of NACT and fast-track recovery procedures for allowing earlier patient mobility, recovery, and hospital discharge for patient not candidate to primary debulking surgery. Accordingly, we found that our comprehensive treatment approach had no significant impact on HRQoL over time in this pioneering trial, with a transitory postoperative impairment quite similar to that we have observed when performing IDS alone.

Despite most of the criticism surrounding to use of HIPEC targets the inherent potential morbidity of a treatment approach combining advanced cytoreductive surgery (CRS), IP chemotherapy and hyperthermia, a recent large US study exploring the use of HIPEC for various peritoneal malignancies indicated this approach is safe when compared with similar-risk major oncologic procedures, and it highlights the presumable high complication rates were a misperception from early CRS/HIPEC experience ${ }^{15}$. Additionally, based on patient-reported outcomes by OV-HIPEC trial' ${ }^{7}$, current data indicate comparable HRQoL and symptom burden between patients treated with IDS plus HIPEC and those peers treated without HIPEC. In line with these findings, a previous systematic review and meta-analysis of 15 studies including 1583 patients also suggested CRS/HIPEC might even confer a small to medium benefits for HRQoL in patients with peritoneal 
carcinomatosis $^{16}$.

Of note, in the aforementioned landmark trial by Van Driel et al. ${ }^{6}$, ostomies were performed more commonly among patients in the surgery-plus-HIPEC group (11\% vs. $17 \%, p=0.19$ ) at a bowel resection rate of $24 \%$ in both study arms, which grossly contrast with the nil rate of ostomy in our study, despite $60 \%$ of our patients requiring a major bowel resection. As previously reported, these ostomies are associated with clinically meaningful and persistent impairments in bowel-related QoL, and related functional domains in patients underwent bowel resection after CRS/HIPEC ${ }^{17}$. Although the restoration of bowel continuity after CRS/HIPEC is successful in most patients, a relatively high complication rate has also been reported ${ }^{18}$. Contrarily, no statistically significant differences were observed between groups over time in the OV-HIPEC trial for the gastro-intestinal complaints, using the EORTC QLQCR38 questionnaire that addressed symptoms relevant for patients undergoing abdominal surgery ${ }^{7}$.

Another main discrepanting findings between OV-HIPEC trial ${ }^{6}$ and our owns is the binomium of longer operative time [338 (299-426) vs. 490 (390-605.8) minutes, respectively] and shorter hospital stay [10 (8-12) vs. 5 (4-6.5) days, respectively] favoring the present study. The duration of surgery is often a reflection of tumor burden and complexity of the procedures that are intrinsically related to increased postoperative morbidity and thus, impaired HRQoL. It is also an indirect measurement of surgical quality and radicality for cancer surgeries. Accordingly, applying current standards of fast-track recovery for advanced CRS in EOC (with or without HIPEC) ${ }^{19,20}$ in association to a shortcourse regimen of HIPEC that only lasts less than one hour in the chemoperfusion itself (data not shown), we were able to enhance postoperative recovery for our study population with a very short length of hospitalization, manageable morbidity and no apparent impairment over time on QoL. If improved survival outcomes can be confirmed after longer follow-up, our comprehensive approach might be explored as an alternative to the standard 90-minutes in length regimen by OV-HIPEC trial ${ }^{6}$, since our all-in-one protocol seems to be more simpler for patient, surgeon, and nursing caregivers.

Our study was limited by the slow accrual that leads us to review our study design and to anticipate the completion of the study. Despite the efforts of other
Brazilian cancer centers to participate in this trial, we experienced the difficulties for developing surgical trials that required major changes in current surgical practices. Further, we were not able to apply additional targeted questionnaires that could help to improve the analysis of QoL after CRS/HIPEC. Instead, we used just the generic EORTC QLQ-C30 questionnaire that may not adequately capture some changes in specific domains of relevance for woman suffering of advanced EOC. Additional limitations include the absence of a baseline QoL measurement just before starting treatment, because our focus was directed to the CRS/HIPEC component of our protocol; as well as the missing data of the last questionnaire from two patents due to disease progression or treatment of postoperative complications and the longer than initially planned time (i.e.: 3-6 week after the last cycle of adjuvant chemotherapy) for collecting these questionnaires, which may have both mitigated our QoL analyses into the context of a small study. On the other hand, we highlight the pioneering of our comprehensive treatment approach involving HIPEC that adds some pieces of evidence to the issue of QoL after HIPEC.

In conclusion, we report a prespecified analysis of the secondary end-point on HRQoL from our pioneering clinical trial exploring the strategy of combining perioperative chemotherapy (i.e.: NACT plus adjuvant chemotherapy), advanced CRS techniques, fast-track recovery procedures and short-course HIPEC for advanced EOC. Accordingly, we found no significant impairment of short-course HIPEC on patient's HRQoL into the context of our all-in-one approach.

\section{ACKNOWLEDGEMENTS}

We would like to acknowledge the Rand Company (Medolla, Italy), for the technical assistance and to all nursing, clinical, surgical and administrative staffs of the involved institutions.

\section{FUNDING}

This study was supported by grants from Decit/ SCTIE/MS - CNPq/FACEPE/SES-PE (APQ:0187-4.01/13) and FAPE/IMIP. 


\section{CONTRIBUTION TO AUTHORSHIP}

Study concept and design: All authors, mainly Batista TP and Lustosa RJC; Acquisition of data: Batista TP, Carneiro VCG, Sarmento BJQ, Lopes A and Costa RLR; Analysis and interpretation of data: Lustosa RJC,
Batista TP, Mello MJG and Lima JTO; Drafting of the manuscript: Lustosa RJC and Batista TP; Critical revision of the manuscript for important intellectual content: All authors, mainly Leão CS, Badiglian-Filho L and Mello MJG; Administrative and material support: Leão CS; Study supervision: Leão CS and Badiglian-Filho L.

\title{
R E S U M O
}

\begin{abstract}
Objetivo: Avaliar o impacto da quimioterapia intraperitoneal hipertérmica (HIPEC) de curta duração (i.e.: 30 minutos) na qualidade de vida (QoL) relacionada à saúde (HRQoL) no contexto de ensaio clínico terapêutico piloto; NCT02249013. Métodos: Avaliou-se o desfecho secundário predeterminado de $H R Q o L$ em ensaio clínico de fase 2 de segurança e eficácia, aberto, multicêntrico, de braço único, utilizando-se o questionário European Organization for Research and Treatment of Cancer Quality of Life (EORTC QLQ-C30, versão 3.0). As pacientes foram solicitadas a responder o questionário de HRQoL antes do tratamento, após a HIPEC, e ao fim do tratamento interdisciplinar. As variações da HRQoL ao longo do tempo foram avaliadas pelas medianas dos escores de cada domínio e analisadas pelo teste de Friedman, considerando-se nível de significância estatística bicaudal de 5\%. Resultados: Quinze pacientes com câncer de ovário de grande volume tumoral foram recrutadas do sistema de saúde pública (i.e.: SUS) entre fevereiro de 2015 e julho 2019. Um questionário basal e pelo menos um questionário de acompanhamento foram coletados de todas as pacientes. Não se observou diferença significativa ao longo do tempo na HRQoL em nenhum dos domínios ou sintomas estudados ( $p>0,05)$. 0 comprometimento transitório da HRQoL imediatamente após a HIPEC de curta duração tendeu a retornar à linha de base ao final do tratamento multimodal. Conclusões: Não se observou impacto significativo da HIPEC de curta duração sobre a HRQoL no contexto deste protocolo de tratamento interdisciplinar.
\end{abstract}

Palavras chave: Injeções Intraperitoneais. Hipertermia Induzida. Tratamento Farmacológico. Neoplasias Peritoneais. Procedimentos Cirúrgicos Operatórios.

\section{REFERENCES}

1. Armstrong DK, Walker JL. Role of Intraperitoneal Therapy in the Initial Management of Ovarian Cancer. J Clin Oncol. 2019 Sep20;37(27):2416-9. doi: 10.1200/JCO.19.00671. Epub 2019 Aug 12.

2. Wright $A A$, Cronin $A$, Milne $D E$, Bookman $M A$, Burger RA, Cohn DE, et al. Use and Effectiveness of Intraperitoneal Chemotherapy for Treatment of Ovarian Cancer. J Clin Oncol. 2015 Sep10;33(26):2841-7. doi: 10.1200/ JCO.2015.61.477.

3. Walker JL, Armstrong DK, Huang HQ, Fowler J, Webster K, Burger RA, et al. Intraperitoneal catheter outcomes in a phase III trial of intravenous versus intraperitoneal chemotherapy in optimal stage III ovarian and primary peritoneal cancer: a Gynecologic Oncology Group Study. Gynecol
Oncol. 2006;100(1):27-32. doi: $10.1016 / j$. ygyno.2005.11.013.

4. Wenzel LB, Huang HQ, Armstrong DK, Walker JL, Cella D; Gynecologic Oncology Group. Healthrelated quality of life during and after intraperitoneal versus intravenous chemotherapy for optimally debulked ovarian cancer: a Gynecologic Oncology Group Study. J Clin Oncol. 2007 Feb1;25(4):437-43. doi: 10.1200/JCO.2006.07.3494.

5. Batista TP, Carneiro VCG, Tancredi R, Teles ALB, Badiglian-Filho L, Leão C. Neoadjuvant chemotherapy followed by fast-track cytoreductive surgery plus short-course hyperthermic intraperitoneal chemotherapy (HIPEC) in advanced ovarian cancer: preliminary results of a promising allin-one approach. Cancer Manag Res. 2017;9:869878. Published 2017 Dec 13. doi:10.2147/CMAR S153327. 
6. Van Driel WJ, Koole SN, Sikorska K, Schagen van Leeuwen JH, Schreuder HWR, Hermans RHM, et al. Hyperthermic Intraperitoneal Chemotherapy in Ovarian Cancer. N Engl J Med. 2018;378(3):23040. doi:10.1056/NEJMoa1708618.

7. Koole SN, Kieffer JM, Sikorska K, Schagen van Leeuwen JH, Schreuder HWR, Hermans RH, et al. Health-related quality of life after interval cytoreductive surgery with or without hyperthermic intraperitoneal chemotherapy (HIPEC ) in patients with stage III ovarian cancer. Eur J Surg Oncol. 2019;:S0748-7983(19)30444-5. doi:10.1016/j. ejso.2019.05.006.

8. Koole SN, van Lieshout C, van Driel WJ, et al. Cost Effectiveness of Interval Cytoreductive Surgery With Hyperthermic Intraperitoneal Chemotherapy in Stage III Ovarian Cancer on the Basis of a Randomized Phase III Trial. J Clin Oncol. 2019;37(23):2041-50. doi:10.1200/JCO.19.00594.

9. Spriggs DR, Zivanovic O. Ovarian Cancer Treatment - Are We Getting Warmer? N Engl J Med. 2018;378(3):293-294. doi: 10.1056/ NEJMe1714556.

10. Vergote I, Harter P, Chiva L. Hyperthermic intraperitoneal chemotherapy does not improve survival in advanced ovarian cancer. Cancer. 2019;125 Suppl 24:4594. doi:10.1002/cncr.32496.

11. Provencher DM, Gallagher CJ, Parulekar WR, Ledermann JA, Armstrong DK, Brundage $M$, et al. OV21/PETROC: a randomized Gynecologic Cancer Intergroup phase II study of intraperitoneal versus intravenous chemotherapy following neoadjuvant chemotherapy and optimal debulking surgery in epithelial ovarian cancer. Ann Oncol. 2018;29(2):431-8. doi:10.1093/annonc/mdx754.

12. Aaronson NK, Ahmedzai S, Bergman B, Bullinger M, Cull A, Duez NJ, et al. The European Organization for Research and Treatment of Cancer QLQ-C30: a quality-of-life instrument for use in international clinical trials in oncology. J Natl Cancer Inst. 1993;85(5):365-76. doi:10.1093/jnci/85.5.365.

13. Tsunoda AT, Ribeiro R, Reis RJ, et al. Surgery in ovarian cancer - Brazilian Society of Surgical Oncology consensus. BJOG. 2018;125(10):12431252. https://doi:10.1111/1471-0528.15328.
14. Tewari D, Java JJ, Salani R, Armstrong DK, Markman $M$, Herzog $T$, et al. Long-term survival advantage and prognostic factors associated with intraperitoneal chemotherapy treatment in advanced ovarian cancer: a gynecologic oncology group study. J Clin Oncol. 2015;33(13):1460-6. doi: 10.1200/ JCO.2014.55.9898.

15. Foster JM, Sleightholm R, Patel A, et al. Morbidity and Mortality Rates Following Cytoreductive Surgery Combined With Hyperthermic Intraperitoneal Chemotherapy Compared With Other HighRisk Surgical Oncology Procedures. JAMA Netw Open. 2019;2(1):e186847. doi: 10.1001/ jamanetworkopen.2018.68.

16. Shan LL, Saxena A, Shan BL, Morris DL. Quality of life after cytoreductive surgery and hyperthermic intra-peritoneal chemotherapy for peritoneal carcinomatosis: A systematic review and metaanalysis. Surg Oncol. 2014;23(4):199-210. doi: 10.1016/j.suronc.2014.10.002. Epub 2014 Oct 28.

17. Bayat Z, Taylor EL, Bischof DA, McCart JA, Govindarajan A. Impairments in Bowel Function, Social Function and Quality of Life After Cytoreductive Surgery and Hyperthermic Intraperitoneal Chemotherapy. Ann Surg Oncol. 2020;27(1):124-131. doi: 10.1245/s10434-01907385-w. Epub 2019 May 9.

18. de Cuba EM, Verwaal VJ, de Hingh IH, van Mens LJ, Nienhuijs SW, Aalbers AG, et al. Morbidity associated with colostomy reversal after cytoreductive surgery and HIPEC. Ann Surg Oncol. 2014;21(3):883-90. doi:10.1245/s10434-013-3370-2.

19. Webb C, Day R, Velazco CS, Pockaj BA, Gray RJ, Stucky CC, et al. Implementation of an Enhanced Recovery After Surgery (ERAS) Program is Associated with Improved Outcomes in Patients Undergoing Cytoreductive Surgery and Hyperthermic Intraperitoneal Chemotherapy. Ann Surg Oncol. 2020;27(1):303-312. doi: 10.1245/s10434-01907900-z. Epub 2019 Oct 11.

20. Lindemann K, Kok PS, Stockler M, Jaaback K, Brand A. Enhanced Recovery After Surgery for Advanced Ovarian Cancer: A Systematic Review of Interventions Trialed. Int J Gynecol Cancer. 2017;27(6):12741282. doi:10.1097/IGC.0000000000000981. 
Received in: 19/03/2020

Accepted for publication: 27/04/2020

Conflict of interest: no.

Funding source: none.
Mailing address:

Thales Paulo Batista

E-mail: t.paulo@outlook.com

(c) (i) 